\title{
Disease Pathogenesis and Research Progression of Renal Anemia
}

\author{
Yingying Liu, Qi Jiang* \\ Department of Nephrology, China-Japan Union Hospital of Jilin University, Changchun, 130033, China \\ *Corresponding Author: Qi Jiang
}

Keywords: Disease pathogenesis, Research progression, Renal anemia

\begin{abstract}
Renal anemia is a common complication of chronic kidney disease (CKD), especially end-stage renal disease. Because of its complex pathogenesis and poor long-term prognosis, it is one of the difficulties in clinical treatment. Erythropoietin deficiency is the main cause of renal anemia. In addition, deficiency of active vitamins, inflammation and infection, and disorder of iron metabolism can further aggravate renal anemia. The treatment of new renal anemia mainly includes two kinds: drug therapy and transfusion therapy. This paper elaborates the pathogenesis and progress of renal anemia to provide references for relevant researchers.
\end{abstract}

\section{Introduction}

Anemia is the most common complication of chronic kidney disease [1]. It is also an important risk factor to promote the progress of chronic diseases and increase the morbidity and mortality of cardiovascular complications. Correcting anemia is of great significance to alleviate symptoms, improve survival rate and quality of life of patients. Renal anemia refers to anemia caused by insufficient erythropoietin production in the kidney, accumulation of toxins in the body and disturbance of internal environment in patients with chronic renal failure, with or without blocked erythropoiesis and shortened life span. Without intervention, with the gradual decline of renal function, patients with CKD will inevitably suffer from hemorrhage and anemia, and the hemoglobin value is positively correlated with GFR in CRF patients. Anemia can lead to hypoxia and cognitive function changes in CKD patients, and seriously reduce the quality of life of CKD patients with long survival time due to improved dialysis technology. Studies have shown that cardiovascular morbidity and mortality in CKD patients increase with the increase of anemia. And now hemodialysis treatment has been quite common, almost all county people's hospitals and Chinese hospitals have carried out hemodialysis treatment, so that the survival rate of uremic patients increased sharply. Therefore, correcting anemia, improving the quality of life of patients with chronic renal failure, reducing the number of hospitalizations or shortening the length of hospitalization, reducing complications, become the main line of treatment for patients with advanced chronic renal failure. Renal anemia is a common and important complication in patients with chronic renal failure. Anemia occurs in many end-stage renal diseases. The main causes are relative or absolute reduction of erythropoietin production, iron deficiency, shortened erythrocyte life span, blood loss, malnutrition, and fibrocystic osteitis caused by secondary hyperparathyroidism. In short, it is the result of a combination of various factors. Renal anemia seriously affects the quality of life of patients, and the process of correcting anemia can also lead to many adverse reactions [2].

\section{Disease Pathogenesis of Renal Anemia}

\subsection{Shortage of EPO.}

Erythropoietin (EPO) is an endogenous glycoprotein hormone that stimulates erythropoiesis. Hypoxia can stimulate the production of erythropoietin. Recombinant human erythropoietin has been used for clinical treatment of anemia associated with renal insufficiency, acquired immunodeficiency 
syndrome or anemia caused by treatment, anemia associated with malignant tumors and rheumatic anemia. Some scholars believe that EPO can play an immunoregulatory role in vivo, inhibit inflammation, alleviate the inhibition of bone marrow hematopoietic system, improve the responsiveness of EPO, thereby affecting hematopoietic function. EPO can also enhance the activity of erythrocyte glutathione peroxidase and superoxide dismutase (SOD) and inhibit the production of oxygen free radicals. It maintains the normal intracellular electrolyte concentration, guarantees the normal osmotic pressure balance inside and outside the membrane, and reduces the osmotic fragility of red blood cells. However, the actual measurement of serum EPO in CRF patients is not low, which may be related to hypoxia, anemia stimulating kidney to produce EPO increase and renal dysfunction reducing blood EPO clearance rate. Hypoxia is the strongest inducing factor, and hypoxia inducing factor (HIF) plays an important role. When oxygen partial pressure drops, HIF, the most important factor for hypoxia, stops degrading and promotes EPO synthesis. The biological effect of EPO on erythropoiesis is realized by the specific EPO receptor located on the surface of bone marrow erythroid progenitor cells. The abnormality of EPOR will lead to anemia. Under the stimulation of EPO, EPOR rapidly produces homologous dimerization, and the formation of homologous dimer can lead to the activation of receptor, which is the physiological effect of EPO. Necessary Conditions Thereafter, EPOR connects the cytoplasmic protein tyrosine kinase, phosphorylates tyrosine, and promotes the proliferation and differentiation of target cells through the second messenger signal transduction pathway.

\subsection{Shortage of Vitamin D.}

Vitamin D is a steroid derivative with anti-rickets effect, also known as rickets resistant vitamin. At present, it is considered that vitamin D is also a steroid hormone. Vitamin D is a fat-soluble vitamin with five compounds, vitamin D2 and vitamin D3 are closely related to health. Vitamin D in vivo, including vitamin D3 synthesized by 7-dehydrocholesterol through skin and vitamin D2 absorbed by food, is hydroxylated into hydroxyvitamin through liver, and then enters epithelial cells through receptor-mediated endocytosis in proximal renal tubules, which is converted into active vitamin under the action of hydroxylase. D. With the gradual decline of renal function in CKD patients, renal parenchyma atrophy leads to the lack of hydroxylase and the production of active vitamin D. In addition, excessive PTH concentration, hyperphosphatemia and hypocalcemia can also inhibit the activation of vitamin D. In addition to promoting calcium ion bone deposition and regulating calcium and phosphorus metabolism, recent studies have found that vitamin $\mathrm{D}$ also participates in regulating a variety of immune metabolism, immune response and inflammatory factors. Inflammatory factors in the circulation of patients receiving maintenance renal replacement therapy decreased significantly after vitamin D3 treatment, and the degree of anemia was improved. This may relieve the inhibition of these inflammatory factors on erythropoietic hematopoietic cells and the effect on EPO responsiveness, which proves the effect of vitamin D on renal anemia from the side. IL-6 also promotes the synthesis of ferritin, which can inhibit the process of intracellular iron outward transport mediated by ferritin transporters, thus affecting iron absorption, tissue distribution and extracellular iron concentration, leading to functional iron deficiency, iron utilization disorder and erythropoietin cannot play its role to the maximum, leading to anemia. The levels of hepcidin and ferritin decreased and transferrin increased [3].

\subsection{Inflammation.}

Although dialysis therapy can remove toxins, it can also remove antioxidants in vivo, making patients more sensitive to oxidative damage and less tolerant, aggravating inflammation. Some patients who use tunnel polyester catheter for hemodialysis and peritoneal dialysis, combined with low internal resistance, are at risk of catheter-related infection or peritonitis, and even sepsis. Clinically, we have noticed that not all patients with renal anemia have a high responsiveness to rh-EPO treatment, and a considerable part of the anemia has not improved significantly. Regular monitoring of renal replacement therapy found that inflammation may affect anemia through the following mechanisms. Animal experiments in vitro showed that IL-1beta, TNF-alpha and TGF-beta 
could inhibit the synthesis of erythropoietin in vitro, while IL-1beta could inhibit the synthesis of EPO in vitro. The membrane of senescent erythrocytes has been changed and cleared by macrophage recognition, which is also the way of clearing normal senescent erythrocytes in vivo. When coated with immunoglobulins or immune complexes, it is easier to be recognized and cleared by macrophages. Inflammatory state in vivo can not only activate macrophages, but also increase the encapsulation on the surface of red blood cells, so that red blood cells are excessive and cleared, reducing the number of red blood cells in vivo. Immune activation of macrophage system strengthens the synthesis of ferritin, reverses the transport pathway, and stores iron in the reticular endothelial system, resulting in functional iron deficiency, which reduces the utilization of iron, resulting in EPO resistance and anemia. Inflammation leads to malnutrition. Studies have shown that inflammation accelerates lipid and protein metabolism, which disappears as inflammation subsides. But when the body of CRF patients is in a persistent state of inflammation for a long time, the body is always in a state of hypermetabolism, which easily leads to malnutrition, thus aggravating anemia [4].

\subsection{Iron Metabolism Disorders.}

Iron metabolism disorder is one of the important factors affecting renal anemia, and ferritin plays an important role in iron metabolism. Ferritin is a small molecule polypeptide hormone synthesized by the liver. As a regulatory protein, it mainly acts on intestinal epithelial cells, liver cells and phagocytes of reticular endothelial cell system to regulate iron metabolism. Ferritin is regulated by intracellular storage of iron and transferrin-bound iron. Elevated serum ferritin levels may occur in excessive iron storage, injury, cancer, inflammatory state or sepsis. In vivo iron excess, inflammation, hypoxia and other conditions will stimulate its synthesis increased, the decline of renal function will also lead to accumulation in the body due to reduced excretion. The up-regulation of ferritin in CKD changes the pathway of iron uptake and transport, hinders iron uptake, hinders iron release from macrophages of reticuloendothelial system, and leads to iron accumulation in reticuloendothelial system, but cannot be used to synthesize hemoglobin. The total iron storage in the patient's body is increased, but the available iron is not much, resulting in disorder of iron metabolism and inadequate raw materials for synthesizing red blood cells. At the same time, calmodulin inactivated transferrin and blocked the output of iron, which increased the dose of ESAs. This kind of iron deficiency not only affects anemia from raw materials, but also iron deficiency is a common cause of EPO resistance. Microinflammation and iron overload are common in CKD patients, which can stimulate the up-regulation of serum ferritin expression, especially in MHD patients with increased risk of infection and inflammation, and the level of ferritin is significantly higher than that in normal people. Excessive levels of ferritin can inhibit the absorption of iron in intestinal tract, release and output of iron in liver and reticular epithelial cell system, and lead to disorder of iron metabolism. Hb synthesis decreased, aggravating the anemia state of CKD patients.

\section{Research Progression of Renal Anemia}

\subsection{Drug Therapy.}

Traditional ESA drugs are combined with EPOR to induce a series of intracellular signal transduction, stimulate the production of red blood cells, maintain the normal life of red blood cells, and improve anemia. If there is a drug, it can bind with EPOR, start a series of hematopoietic and erythrocyte protection mechanisms, but the molecular structure and EPO do not have homology, avoid the production of anti-EPO antibodies; at the same time, it can avoid intravenous administration, instead of routine oral administration, reduce injection pain, improve patients' compliance and quality of treatment, better. The treatment of renal anemia. In recent years, EPO mimetic peptides with the above characteristics have become the focus of research, and no anti-EPO neutralizing antibody has been found in such studies. Patients with chronic kidney disease, whether dialysis or non-dialysis, should be supplemented with iron to achieve and maintain the target value of iron status. Intravenous iron supplementation is the best way to supplement iron. Sucrose iron is the 
safest target value of intravenous iron supplementation. During erythropoietin treatment, adequate iron supplementation is needed. Hemodialysis patients: serum ferritin $>200 \mathrm{gn} / \mathrm{mL}$; non-hemodialysis patients: serum ferritin $>100 \mathrm{ngm} / \mathrm{L}$; hemodialysis patients preferred intravenous injection, non-dialysis patients can either intravenous injection, or oral iron. According to the patient's body weight, hemoglobin target value, actual value, different patients choose different doses of and erythropoietin-like. Like peptides, HIF stabilizers are administered orally, and the range of drug concentration fluctuation is safer than that of injections, which also greatly improves patient compliance. It stabilizes plasma EPO in physiological range and avoids the increase of plasma EPO hyper physiological state caused by ESAs intravenous application. In addition, it can inhibit the expression of ferritin and regulate iron. The distribution and absorption of iron in the body promote the utilization of iron.

\subsection{Transfusion Therapy.}

In patients with chronic anemia, red blood cells need not be transfused if the hemoglobin level is high enough to prevent tissue hypoxia and heart failure. If red blood cells are transfused as a long-term treatment, the target value of hemoglobin should be determined in advance and evaluated continuously during the treatment. Long-term massive blood transfusion can lead to iron overload. When the hemoglobin concentration of patients with chronic renal insufficiency has reached the standard, no blood transfusion is needed. Blood transfusion is required only in emergencies or when hemoglobin is rapidly declining, and international guidelines have set standards for when transfusion therapy is needed. However, active iron replacement therapy can largely avoid blood transfusion, even for hemodialysis patients. Blood transfusion should be avoided as far as possible in the treatment of renal anemia, especially in patients expecting kidney transplantation. Hemodialysis patients suffer from hemorrhage during hemodialysis, frequent blood test and uremia patients often accompanied by bleeding tendency, which can lead to further aggravation of anemia. Therefore, reducing blood loss during dialysis, avoiding long-term frequent blood test and avoiding bleeding tendency are important factors to improve anemia. Regular hemodialysis, clearance of blood toxins, alleviation of the inhibition of toxins on bone marrow and reduction of erythrocyte damage are also important to improve anemia in patients with chronic renal insufficiency. CKD anemia patients have long tolerated low levels of $\mathrm{Hb}$ in vivo, so it is impossible to judge whether patients should be treated with blood transfusion solely based on the level of $\mathrm{Hb}$. Blood transfusion should be considered only when patients are unable to tolerate the dysfunction caused by anemia, such as heart failure, acute blood loss, severe anemia, or perioperative management. Generally, hemoglobin $<60 \mathrm{~g} / \mathrm{L}$, blood transfusion therapy should be considered. However, if the patients with COPD, coronary heart disease and old age have poor tolerance to anemia, they can be relaxed appropriately.

\section{Conclusion}

At present, the treatment of renal anemia is mostly achieved by supplementing EPO, iron and blood transfusion when severe anemia occurs. There are many uniform treatments and lack of individualized and targeted treatment. Through this review, the aim is to sort out the mechanism and treatment of renal anemia, update the latest research in time, implement individualized treatment for patients, improve the degree of anemia to the greatest extent, and improve the long-term survival rate and quality of life of CRF patients with continuous improvement of dialysis technology.

\section{References}

[1] Zhou J, Guo F, Duan S, et al. Clinical Observation of Ferrous Succinate versus Shengxuening in the Adjuvant Treatment of Renal Anemia in Patients with Diabetic Nephropathy Peritoneal Dialysis[J]. China Pharmacy, 2016, 27(27): 3777-3779.

[2] Xu D Y, Shen L, Zhou L, et al. Correlation of serum vitamin C content with renal anemia, oxidative stress and microinflammatory state in patients with peritoneal dialysis[J]. Journal of Hainan 
Medical University, 2017, 23(18): 57-60.

[3] Dai X, Cai S. Application Value of Multi Parameter Analysis of Net and Red Blood Cells in the Treatment of Renal Anemia[J]. Journal of Modern Laboratory Medicine, 2017, 32(2): 146-148+152.

[4] Cases A, Egocheaga M I, Tranche S, et al. Anemia of chronic kidney disease: Protocol of study, management and referral to Nephrology[J]. Nefrología (English Edition), 2018, 38(1): 8-12. 